\title{
Transplantation of nucleoli into human zygotes: not as simple as expected?
}

\author{
Josef Fulka Jr • Alena Langerova • Pasqualino Loi • \\ Stanislava Martinkova $\cdot$ Helena Fulka
}

Received: 1 February 2011 /Accepted: 29 March 2011/Published online: 8 April 2011

(C) Springer Science+Business Media, LLC 2011

\section{Introduction}

Mammalian oocytes and one-cell stage embryos contain prominent nuclear organelles, the nucleolus precursor bodies (nucleoli), that are structurally and functionally different when compared to nucleoli in more developmentally advanced embryos and somatic cells. The function of these organelles, called the nucleolus precursor body$\mathrm{NPB}(\mathrm{s})$, is not known and it is commonly accepted that they might serve as the storage site of material from which fully functional nucleoli are gradually formed. When evaluated ultrastructurally, NPBs are exclusively composed from dense fibrillar material. On the other hand, fully differentiated nucleoli contain dense fibrillar, fibrillar and granular components and are involved in many cellular processes, i.e. transcription of ribosomal genes, ribosome biogenesis, cell cycle progression and proliferation, gene silencing and so on. The differentiated nucleolus contains

Capsule Development of one-cell stage human embryos with low number of nucleoli and their unequal distribution in pronuclei is compromised. Thus, it has been suggested that the transplantation of extra-nucleolar material may possibly rescue these embryos. Recently published results, however, show that this might be more complicated than expected.

\footnotetext{
J. Fulka Jr $(\bowtie) \cdot$ S. Martinkova $\cdot$ H. Fulka

Institute of Animal Science,

Pratelstvi 815,

CS-104 00 Prague 10, Czech Republic

e-mail: fulka@vuzv.cz
}

\author{
A. Langerova \\ GENNET, \\ Prague, Czech Republic \\ P. Loi \\ University of Teramo, \\ Teramo, Italy
}

about 300 proteins [1]. The composition of NPBs is unknown - the only protein that has been demonstrated to localize to NPBs without any doubts is nucleoplasmin 2 [2].

Nucleoli (NPBs) are well visible in growing and fully grown oocytes of some mammals, i.e. mouse, rat, pig human, etc. On the other hand, they cannot be observed for example in native bovine, sheep and rabbit oocytes. In most developmentally advanced fully grown oocytes, that are competent to undergo germinal vesicle breakdown and reach metaphase II stage, nucleoli are surrounded with a ring of chromatin. In less developed oocytes, the chromatin is dispersed in the nucleoplasm [3]. Similar chromatin attachment can be seen also in pronuclei. It is not fully and completely understood why contact of NPB/chromatin is so important from the developmental point of view.

From a human assisted reproduction point of view an interesting observation has been made by Tesarik and coworkers [4]. These authors found that the pattern of distribution of nucleoli as well as their number in pronuclei of one-cell stage human embryos reflect developmental potential of these embryos and can be used as a noninvasive tool for selecting the best embryos. These observations were confirmed by others. But again, why zygotes with various patterns differ developmentally and why some of them have a certain pattern and in others the pattern is different remains to be explained. Evidently, these results however indicate the importance of nucleoli even in one cell stage embryo. This has been confirmed by Burns et al. [5] who produced knock-out NPM2 (nucleoplasmin 2) mice. Nucleoplasmin 2 is important in Xenopus as it helps to decondense sperm DNA. In contrast, it does not play the same role in the mouse. In NPM $2^{-/-}$mice the sperm head decondenses in the oocyte cytoplasm but in pronuclei no nucleoli can be detected. Development of these embryos is compromised and is typically arrested at the two-cell stage. 
Nevertheless, some knock-out female mice gave birth to offspring. Interestingly, the typical nucleoli cannot be seen in immature $N P M 2^{-/}$oocytes, but surprisingly, their maturation was apparently normal and they reached metaphase II stage.

\section{Manipulating the mammalian oocyte nucleoli}

In 2003 Fulka, Jr. et al. reported that the nucleolus can be removed from fully grown porcine oocytes by micromanipulation - the enucleolation [6]. The same approach has been shown (enucleolation) to work well in the mouse too and probably in some others mammals where oocyte nucleoli are clearly visible [7, 8]. Nucleoli can also be removed from pronuclei of one-cell stage embryos (Figs. 1a-d). Here, however, the method is more complicated and the complete enucleolation is only successful when each pronucleus contains just a single nucleolus. The enucleolation thus gave us an unrivalled opportunity to study the function of NPBs in more detail. Thus, it has been showed that oocyte nucleoli are not essential for the initiation and completion of oocyte maturation. The manipulated and control oocytes reached metaphase II at the same frequency. The enucleolated oocytes can be parthenogenetically activated or fertilized but their pronuclei do not contain the typical nucleoli and the resulting embryos usually do not cleave beyond the two-cell stage. As expected, the detailed analysis of these embryos indicated defects in transcription. The embryo nucleolus is thus of maternal (oocyte) origin $[9,10]$.

An interesting technical observation has been made by Fulka and Fulka, Jr. who found that if the nucleolus (NPB) is transferred into the oocytes (zygote) cytoplasm it is rapidly translocated into the nucleus-germinal vesicle (GV) - or into pronuclei (PNs). Thus, it is not necessary to inject the nucleoli directly into nuclei and this greatly facilitates the micromanipulation procedure and largely reduces the oocyte or zygote damage. Therefore, the authors suggested that it might be possible, by simple injection, to rescue human zygotes having an abnormal pattern or number of nucleoli in PNs [11]. The recently published papers however showed that this will be more complicated than expected.

\section{Nucleoli in oocytes and one-cell stage embryos}

As pointed out earlier, the NPBs play an important structural role both in oocytes and in zygotes by helping to organize the chromatin in these cells.

Recently, several experiments demonstrated that in zygotes, especially the pericentric heterochromatin is associated with the NPBs. This association is typical for transcriptionally inactive embryonic stages. However, upon the activation of the embryonic genome the centromeres are re-localised from NPBs to chromocenters that are typical for somatic cells. This process is probably important for the functional maturation of centromeres and pericentric heterochromatin. This, in turn, is necessary for example for the correct segregation of chromosomes during subsequent cell divisions and in consequence, for embryonic development $[12,13]$.

It has been documented that after fertilization, the sperm chromatin, that is originally organized mostly by protamines, undergoes decondensation and protamines are gradually replaced by histones originating from the oocyte cytoplasm. As a result, the epigenetic information carried by covalent histone modifications must be newly set. In contrast, the oocyte does not exhibit extensive replacement of histones (except for the linker histone, for review see [14]). Therefore we might expect the parental genomes to be epigenetically unequal shortly after fertilization. Indeed this had been extensively documented in the past years both on the whole genome scale and also specifically for centric and pericentric chromatin. Probst and colleagues [15] showed that while the female pronucleus exhibits the presence of histone modifications typical for centric and pericentic heterochromatin ( $\mathrm{H} 3 \mathrm{~K} 9 \mathrm{me} 3, \mathrm{H} 3 \mathrm{~K} 27 \mathrm{me} 3$ and H4K20me3), these modifications are absent from the male pronucleus. Even at the two-cell embryonic stage, the paternal pericentric chromatin is devoid of $\mathrm{H} 3 \mathrm{~K} 9 \mathrm{me} 3$ [16].

Interestingly, irrespectively of this epigenetic difference between the male and female pronucleus, in both pronuclei the pericentric heterochromatin has been shown to gradually associate with the NPB as it forms $[15,16]$. However, at the two-cell stage, this distribution is lost and the centromeres do no longer associate with the NPBs, instead they become gradually organized to chromocentres - an organization typical for somatic cells [16]. Probst and colleagues [17] has shown that this re-localization into chromocentres is dependent upon transcription of the major satellite repeats. These authors show that in the mouse embryos the transcription of these repeats starts at late zygotic stage and that these repeats are mainly derived from the paternal genome. This is followed by a burst of transcription of these sequences at the time of major genome activation (in the mouse at the two-cell stage) with its subsequent down-regulation. Interestingly, further analysis showed that this process is parent-specific with the forward major satellite transcript mainly originating from the paternal genome. Finally, these authors show that interference with this process leads to developmental arrest.

This observation was confirmed by two more recent articles. In the first one, Santenard and colleagues [12] show that histone H3.3 plays a major role in pericentric 

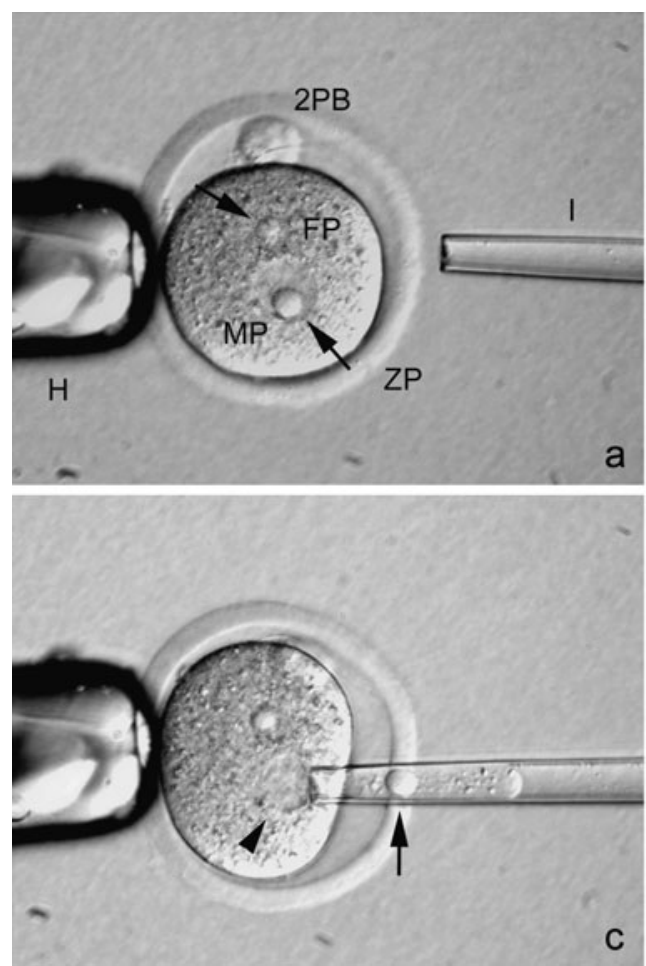

Fig. 1 a-d The enucleolation of one-cell stage mouse embryo. a Onecell pronuclear stage embryo where each pronucleus contains just a single nucleolus is stabilized with a holding pipette $(\mathrm{H})$. The enucleolation ( $\mathrm{I}$-injection pipette is on the right. $\mathrm{ZP}$ - zona pellucida, $2 \mathrm{~PB}$ - second polar body, $\mathrm{FP}$ - female pronucleus (maternal), MPmale pronucleus (paternal), arrows - nucleoli. b The injection pipette first penetrates through zona pellucida and its tip is pushed into a close vicinity of the pronucleus from which the nucleolus has to be removed. Thereafter a very gentle suction is applied. c This mild suction preferentially aspirates the nucleolus that penetrates the pronuclear membrane and moves into the embryo cytoplasm (arrow). In the pronucleus no nucleolus can be seen (arrowhead). Note, that the

heterochromatin formation. When a mutation is introduced at the position 27 (Lysine) of histone H3.3, embryos show aberrant transcription of the pericentric sequences leading to errors in chromosome segregation and finally to developmental arrest. Interestingly, a similar observation was made by Baumann and colleagues who showed that maternal loss of alpha thalassemia/mental retardation X-linked syndrome (ATRX) protein leads to abnormal chromosome morphology and severe reduction of fertility. It was shown that ATRX, that belongs to the SNF2-family remodeling complexes, together with Fas death domain-associated protein (DAXX) localize to the pericentric chromatin domains during oocyte prophase I arrest. Furthermore, the authors show that maternal loss of ATRX abrogates DAXX localization and leads to centromeric and chromosomal instability. What might be the link between these observations?

ATRX and DAXX have previously been shown to localize to telomeres in embryonic stem cells. Moreover, newer results show that ATRX-DAXX complex is requited for the deposition of H3.3 at telomeres [18]. Therefore, one

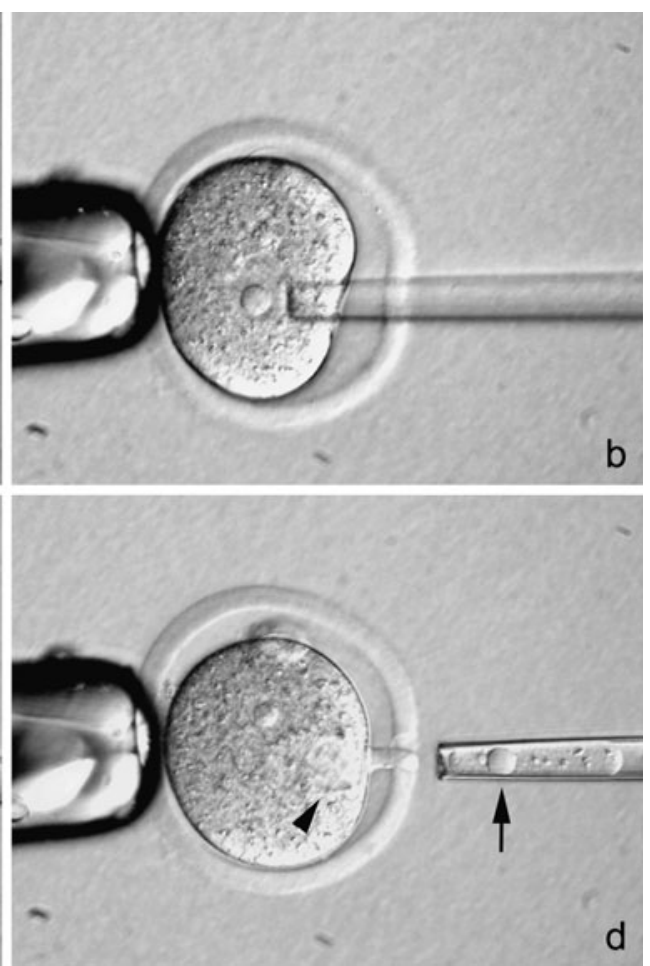

injection pipette actually did not penetrate directly into the embryo cytoplasm. d By increasing the distance between both pipettes, the nucleolus enclosed with an embryo membrane and a minimum volume cytoplasm - arrow (nucleoloplast) can be completely removed from the embryo. Arrowhead - the enucleolated paternal pronucleus. Those embryos from which nucleoli were removed from both pronuclei do not develop beyond the 2-4 cell stage. The enucleolation of either maternal or paternal pronucleus resulted in compromised embryonic development with a minimum of abortive blastocysts produced. The nucleolus can be removed similarly from immature (GV-stage) oocytes

might imagine that if ATRX and DAXX localize to pericentric chromatin in prophase I arrested oocytes they might carry out similar process - that is the deposition of H3.3 histone. H3.3 (and specifically H3.3 K27) in turn seems to be important for the transcriptional regulation of these repetitive sequences [13]. Finally, interfering with this process will lead to non-functional centromeres and pericentric heterochromatin and thus to abnormal and unstable chromosomes and developmental arrest.

Thus to summarize, the above mentioned articles show that the association of centric/pericentric repeats with the NPB might reflect a functional as well as epigenetic "maturation" of these sequences.

\section{Transplanting the nucleolus into one cell stage embryos}

An interesting observation was made by Ogushi and Saitou in 2010 [19]. These authors have transplanted the NPBs from GV stage oocytes into different oocyte maturation 
stages or one-cell stage embryos and examined the development of such manipulated oocytes or embryos. When fertilized, the enucleolated and NPB re-transplanted oocytes at the GV or metaphase II stage had the ability to develop up to the blastocyst stage, on the other hand, when the NPB was re-injected into one-cell stage embryos, originating from previously enucleolated oocytes, the developmental ability of these embryos was severely reduced. These experiments show that the NPB might undergo progressive changes in composition and/or function between the oocyte (GV) and zygote stage. Thus, it is possible that NPBs originating either from oocytes or zygotes are not equivalent albeit they have seemingly identical structure and appearance. The other possibility is that NPBs start to function very soon after fertilization and their transplantation into more advanced zygotes does not allow them to fulfill their typical role(s).

The detailed analysis of higher order chromatin showed that the NPB is the major organizing structure both in oocytes and in early embryos. Thus, the higher order chromatin structure of zygotes originating from enucleolated oocytes showed severe defects in organization (especially with respect of centromeres) although the global level of several histone modifications, that were investigated, was apparently not altered. These findings are not surprising taking into account the recently published articles.

Interestingly, Ogushi and Saitou [19] also show alteration of duration of the first mitotic cell cycle in embryos derived from enucleolated oocytes. Whether this observation reflects defects in centromere and pericentric heterochromatin establishment and function is currently unknown. As pointed out above, two recently published articles highlight the role of epigenetic mechanism operating in establishment of functional centromeres and pericentric heterochromatin in oocytes and early embryos [12, 13]. However, in somatic cells, the nucleolus is also known to play an important role in cell cycle progression by sequestering certain cell cycle involved proteins therefore the explanation for the observed lag in development to the two-cell stage might be the absence of some protein that localizes to the NPB. Unfortunately, as pointed out earlier the protein composition of NPBs is currently unknown and due to technical limitations will be very difficult to establish.

\section{Summary}

To summarize, it is clear that NPBs play an important structural and chromatin organizing role, especially the pericentric heterochromatin and centromeres that associate with this nuclear body. Recent results demonstrate the role of epigenetic mechanisms in establishment of pericentric heterochromatin and centromere function. However, as the protein composition of this unique structure is unknown it is also not known to what extent NPBs play an active role in this process. Ogushi and Saitou [19] have demonstrated that zygotic and GV-stage oocyte NPBs are not equivalent in composition and/or function. Exchanging zygotic NPBs for oocyte NPBs in mouse onecell stage embryos lead to a lag in cell cycle progression and severe developmental arrest. These experiments clearly demonstrate that the proposed nucleolus transplantation therapy will not be trivial. In other words, the mentioned experiments indicate that during oocyte growth and early embryonic development the composition and/or function of the NPB changes, even though the NPB appears morphologically equivalent in these cells. These subtle changes might therefore prevent the proposed micromanipulation therapy (exchange of NPBs between oocyte and zygote or transfer of additional NPB material originating from oocytes to zygotes).

Acknowledgements JFJr's lab is supported from MZe 0002701401 and GACR 523/09/1878, HF is supported from GACR P302/11/P069. PL research is partially funded by NextGene, VII FP, project n.244356.

\section{References}

1. Boisvert FM, van Koningsbruggen S, Navascues J, Lamond AI. The multifunctional nucleolus. Nat Rev Mol Cell Biol. 2007;8:574-94

2. Inoue A, Aoki F. Role of the nucleoplasmin $2 \mathrm{C}$-terminal domain in the formation of nucleolus-like bodies in mouse oocytes. FASEB J. 2010;24:485-94.

3. Wickramasinghe D, Ebert KM, Albertini DF. Meiotic competence acquisition is associated with the appearance of M-phase characteristics in growing mouse oocytes. Dev Biol. 1991;143:162-72.

4. Tesarik J, Junca AM, Hazout A, Aubriot FX, Cohen-Bacrie P, Dumont-Hassan M. Embryos with high implantation potential after intracytoplasmic sperm injection can be recognized by simple, non invasive examination of PN morphology. Hum Reprod. 2000;17:184-7.

5. Burns KH, Viveiros MM, Ren Y, Wang P, DeMayo FJ, Frail DE, et al. Roles of NPM2 in chromatin and nucleolar organization in oocytes and embryos. Science. 2003;300:633-6.

6. Fulka Jr J, Moor RM, Loi P, Fulka J. Enucleolation of porcine oocytes. Theriogenology. 2003;59:1879-85.

7. Fulka H, Mrazek M, Fulka Jr J. Nucleolar dysfunction may be associated with infertility in humans. Fertil Steril. 2004;82: 486-7.

8. Mohammed AA, Karasiewicz J, Modlinski J. Developmental potential of selectively enucleated immature mouse oocytes upon nuclear transfer. Mol Reprod Dev. 2008;75:1269-80.

9. Ogushi S, Palmieri C, Fulka H, Saitou M, Miyano T, Fulka Jr J. The maternal nucleolus is essential for early embryonic development in mammals. Science. 2008;319:613-6. 
10. Lefevre B. The nucleolus of the maternal gamete is essential for life. BioEssays. 2008;30:613-6.

11. Fulka H, Fulka Jr J. Nucleolar transplantation in oocytes and zygotes: challenges for further research. Mol Hum Reprod. 2010;16:63-7.

12. Santenard A, Ziegler-Birling C, Koch M, Tora L, Bannister AJ, Torres-Padilla ME. Heterochromatin formation in the mouse embryo requires critical residues of the histone variant H3.3. Nat Cell Biol. 2010;12:853-62.

13. Baumann C, Viveiros MM, De La Fuente R. Loss of maternal ATRX results in centromere instability and aneuploidy in the mammalian oocyte and preimplantation embryo. PLoS Genet. 2010;6:e1001137.

14. Hajkova P. Epigenetic reprogramming-taking a lesson from the embryo. Curr Opin Cell Biol. 2010;22:342-50.

15. Probst AV, Santos F, Reik W, Almouzni G, Dean W. Structural differences in centromeric heterochromatin are spatially recon- ciled on fertilization in mouse zygotes. Chromosoma. 2007;116:403-15.

16. Merico V, Barbieri J, Zuccotti M, Joffe B, Cremer T, Redi CA, et al. Epigenomic differentiation in mouse preimplantation nuclei of biparental, parthenote and cloned embryos. Chromosome Res. 2007;15:341-60.

17. Probst AV, Okamoto I, Casanova M, El Marju F, Le Baccon P, Almouzni G. A strand specific burst in transcription of pericentric satellites is required for chromocenter formation and early mouse development. Dev Cell. 2010;19:625-38.

18. Lewis PW, Elsaesser SJ, Noh KM, Stadler SC, Allis CD. Daxx is an H3.3-specific histone chaperone and cooperates with ATRX in replication-independent chromatin assembly at telomeres. Proc Natl Acad Sci USA. 2010;107:14075-80.

19. Ogushi S, Saitou M. The nucleolus in the mouse oocyte is required for the early step of both female and male pronucleus organization. J Reprod Dev. 2010;56:495-501. 